\title{
LA NOCTURIA EN PACIENTES CON SÍNTOMAS DE VEJIGA HIPERACTIVA
}

\author{
Montserrat Espuña-Pons', Pedro Blasco², Maite Pérez y y Pablo Rebollo4 .
}

IInstituto Clínico de Ginecología y Obstetricia (ICGON), Hospital Clínico. Barcelona. España.

${ }^{2}$ Servicio de Urología. Hospital de Valme. Sevilla. España.

${ }^{3}$ Departamento Médico. Laboratorios Almirall S.A. Barcelona. España.

${ }^{4}$ BAP Health Outcomes Research S.L. Oviedo. España.

Resumen.- OBJETIVO: La nocturia es un síntoma urinario común en pacientes con vejiga hiperactiva (VH) normalmente asociado a un importante impacto en la calidad de vida. El presente estudio se realizó con el objetivo de describir el perfil del paciente con VH que padece nocturia y así contribuir a la adecuada identificación y manejo de estos pacientes.

MÉTODO: Estudio epidemiológico observacional, multicéntrico y de ámbito nacional de 1454 pacientes mayores de 18 años con diagnóstico previo de VH. Se analizó la relación de la nocturia con diferentes va-

\section{CORRESPONDENCIA}

Montserrat Espuña Pons

ICGON. Hospital Clinic i Provincial C/Villarroel 170

08036 Barcelona (España)

\section{2mep@comb.es}

Aceptado para publicar: 1 de febrero 2010 riables personales y clínicas y se realizaron modelos de regresión logística para identificar las variables asociadas de manera independiente a la nocturia, tanto en mujeres como en varones.

RESULTADOS: El 84,6\% de la muestra presentó nocturia considerando el criterio estricto de dos o más micciones nocturnas (96,8\% según la definición de la ICS). Los sintomas urinarios de frecuencia, urgencia e incontinencia urinaria de urgencia se asociaron de forma bivariada con la nocturia en ambos sexos, mientras que la variable "dolor" se asoció sólo en mujeres y la variable de dificultad miccional "fuerza" sólo en varones. La mayor edad y la presencia de comorbilidades se asocian, tanto de forma bivariada como independientemente en modelos de regresión logística, a la presencia de nocturia en pacientes con $\mathrm{VH}$ de ambos sexos.

CONCLUSIONES: En este estudio se ha determinado que el perfil de los sujetos previamente diagnosticados de $\mathrm{VH}$ que presentan nocturia responde a una mayor edad y a la presencia de comorbilidades de cierta importancia para ambos sexos. El perfil de pacientes con nocturia y $\mathrm{VH}$ presentado difiere de los reportados previamente para pacientes con nocturia y otras patologías urinarias.

Palabras clave: Nocturia. Vejiga hiperactiva. Epidemiología. Síntomas urinarios. Comorbilidad.

Summary.- OBJECTIVES: Nocturia is a frequent urinary symptom in overactive bladder (OAB) patients, which usually exerts a negative impact upon their quality of life. This study describes the profile of the $O A B$ patient with nocturia, in order to contribute to the adequate identification and medical management of these subjects. 
METHODS: Multicenter, national observational study of 1454 patients over 18 years of age, previously diagnosed with $O A B$. The relationship between nocturia and different personal and clinical variables was studied, and logistic regression models were used to identify variables independently associated with nocturia in both women and men.

RESULTS: $84.6 \%$ of the patients presented nocturia, considering the restrictive criterion of two or more micturition episodes per night $196.8 \%$ according to the ICS definition). Urinary symptoms of frequency, urgency and urge incontinence were associated in a bivariate analysis with nocturia for both genders, while the voiding difficulty variable "pain" was only associated in women, and the voiding difficulty variable "straining" only in men. Older age and presence of comorbidities were associated both in bivariate analysis and independently in logistic regression models to the presence of nocturia in $O A B$ patients of both genders.

CONCLUSIONS: This study revealed that the profile of subjects with previously diagnosed $O A B$ and nocturia corresponded to older age and the presence of comorbidities of a certain impact for both men and women. The profile for $O A B$ patients with nocturia differs from those previously reported for patients suffering from nocturia and other urinary pathologies.

Keywords: Nocturia. Overactive bladder. Epidemiology. Urinary symptoms. Comorbidity.

\section{INTRODUCCIÓN}

El "Síndrome de Vejiga Hiperactiva (VH)" se define como la "urgencia", con o sin incontinencia urinaria (IU) de urgencia, a menudo asociada a aumento de frecuencia y/o nocturia (1). La prevalencia de $\mathrm{VH}$ ajustada a la población española se ha situado en un $19,9 \%$ (IC $95 \%$ 18,0-21,8), siendo superior entre las mujeres $(23,6 \%)$ que en los hombres $(15,4 \%)(2)$. Una proporción considerable de pacientes que presentan $\mathrm{VH}$ refieren que se despiertan por la noche debido a la necesidad de orinar.

La International Continente Society (ICS) define la nocturia como síntoma cuando el individuo se queja de que se despierta durante la noche una - más veces para orinar (3). La necesidad de levantarse durante las horas de sueño para ir a orinar es un problema que afecta a hombres y mujeres. La frecuencia miccional nocturna es la necesidad de orinar una o más veces durante la noche en el tiempo comprendido desde que el individuo se acuesta con la intención de dormir hasta el momento del despertar con la intención de levantarse. La frecuencia nocturna se diferencia de la nocturia, en que aquélla incluye las micciones que se realizan después de que el individuo se haya acostado pero antes de haberse quedado dormido, y las micciones de la madrugada si se despierta y no puede volver a dormirse como desearía. La nocturia se diferencia en que considera solamente el tiempo en que el individuo está durmiendo (4).

La prevalencia de la nocturia en la población general es difícil de precisar ya que hay pocos estudios epidemiológicos sobre este problema y además se han utilizado distintas terminologías. Un estudio poblacional realizado con 5.204 personas con una media de edad de 45,8 años, encontró que un $31 \%$ referían levantarse al menos una vez por la noche a orinar y el 14,2\% se levantaba dos veces (5). En dos estudios en los que se ha utilizado como definición de nocturia el tener que levantarse a orinar 2 o más veces, la prevalencia fue de $28,5 \%$ y $11,3 \%$ respectivamente $(6,7)$. Los estudios llevados a cabo hasta el momento coinciden en que la prevalencia de la nocturia aumenta con la edad y que es igual en hombres que en mujeres (8-11).

Aunque un episodio de nocturia puede ser considerado normal en personas sanas y sin otros síntomas urinarios $(12,13)$, cuando este síntoma se produce en pacientes con $\mathrm{VH}$, aunque sólo refieran un solo episodio de nocturia, el 19\% de los hombres y el $24 \%$ de las mujeres afirman que les resulta muy molesto. La proporción de personas con $\mathrm{VH}$ que refieren molestia por la nocturia aumenta cuando refieren que se despiertan dos o más veces. La evaluación de la calidad de vida de los pacientes con $\mathrm{VH}$ mediante cuestionarios validados demuestra cómo la presencia del síntoma nocturia aumenta el impacto que esta patología produce en la calidad de vida, fundamentalmente por su afectación sobre la calidad del sueño (14).

El presente estudio se realizó con el objetivo de describir el perfil del paciente con síndrome de $\mathrm{VH}$ que padece el síntoma nocturia y estudiar la asociación de diferentes variables del paciente con la presencia del síntoma nocturia.

\section{SUJETOS Y MÉTODO}

Estudio epidemiológico observacional, multicéntrico y de ámbito nacional de 1.454 pacientes mayores de 18 años con síntomas de vejiga hiperactiva. La recogida de datos se llevó a cabo en diferentes consultas de Urología y Ginecología entre enero y abril de 2008. 
Se recogieron datos antropométricos de los pacientes (edad, talla peso y perímetro de cintura), así como datos socio-demográficos (sexo, estado civil, raza, y educación), y, en el caso de las mujeres, una serie de preguntas sobre la paridad, la menopausia, los años desde la aparición de la misma y el seguimiento de tratamientos hormonales sustitutivos sistémicos o con estrógenos vaginales.

Todos los pacientes fueron interrogados directamente sobre sus síntomas urinarios siguiendo un cuestionario estructurado (Tabla I). Las preguntas del cuestionario fueron seleccionadas a partir de dos cuestionarios de síntomas, ampliamente utilizados y recomendados para la investigación de síntomas del tracto urinario inferior en mujeres y hombres $(15,16)$. Se recogieron además el número de episodios de nocturia y el grado de comorbilidad mediante el índice de Charlson (Tabla II) (17).

Los datos y valoraciones facilitados por cada paciente e investigador se recogieron por medio de un cuaderno de recogida de datos electrónico. El tratamiento estadístico de los datos se realizó mediante el software SPSS 14 (SPSS Inc., Chicago, Illinois) para Windows.

Las variables continuas consideradas en el análisis se describieron utilizando como medida de la tendencia central su media y como medidas de dispersión su máximo y mínimo y su desviación típica. Las variables categóricas, categorizadas y ordinales se describieron a partir de sus frecuencias absolutas y relativas. Los síntomas urinarios fueron definidos en función de la respuesta de los sujetos a las diferentes preguntas de la Tabla I. Para cada pregunta había 5 opciones de respuesta excepto para las preguntas 3,12 y 15 que había 4 opciones. Se consideraron como positivos aquellos síntomas con una puntuación mayor o igual a 3 . El índice de Charlson fue recodificado para el análisis en tres niveles, según los pacientes presentaran un índice 0,1 o igual o superior a 2. En la Tabla ll se presenta la escala de patologías recogida en dicho índice.

Se estudió la relación de la nocturia según la frecuencia de interrupción del sueño para orinar definida por la ICS (1 o más veces) y según un criterio más estricto comúnmente usado en investigación $(2$ o más veces), con diferentes variables personales y clínicas para los subconjuntos de varones y mujeres.

Para el estudio de la asociación con variables categóricas, categorizadas $u$ ordinales se utilizaron tablas de contingencia y el estadístico Chi cuadrado (con la corrección de Yates para variables dicotómicas), mientras que para las variables continuas estudiadas se empleó la prueba $U$ de MannWhitney una vez comprobado que su distribución en la población no era normal. Por último con aquellas variables personales o clínicas no urinarias que en el análisis bivariado habían presentado asociación estadísticamente significativa con la presencia de nocturia se construyeron modelos de regresión logística por sexos (método de entrada en un solo paso).

\section{TABLA I. CUESTIONARIO DE SÍNTOMAS URINARIOS.}

1. ¿ंTiene que correr al baño para orinar?

2. ¿Con qué frecuencia acude al baño a orinar?

3. ¿le cuesta empezar a orinar?

4. $\dot{2}$ Tiene que hacer fuerza para orinar?

5. ¿ Se le interrumpe el chorro de orina mientras está orinando?

6. ¿Se le escapa la orina cuando siente un fuerte deseo de orinar, difícil de controlar?

7. ¿̇Se le escapa la orina cuando realiza una actividad física como toser, estornudar, reír o correr?

8. ¿̇iente dolor en la vejiga? 


\section{RESULTADOS}

En las Tablas III y IV se presenta la descripción del conjunto de la muestra estudiada (1.454 pacientes con síntomas de Vejiga Hiperactiva) y por sexos (1.056 mujeres, 397 varones) en cuanto a variables demográficas y clínicas generales y a síntomas urinarios respectivamente. Según la codificación del Índice de Charlson, las patologías más frecuentes en la muestra estudiada fueron "diabetes sin lesión órganos diana", 23,2\% (I.C.95\% 21,1 - 25,3), "úlcera gastro-duodenal", 11,5\% (I.C.95\% $9,9-13,2)$ y "enfermedad arterial periférica", $11,1 \%$ (I.C. $95 \% 9,6$ - 12,9). Como se puede comprobar el $84,6 \%$ de la muestra total presentó nocturia considerando el criterio estricto de dos o más micciones nocturnas. Este porcentaje se eleva al 96,8\% según la definición de la ICS. Además un

\section{TABLA II. INNDICE DE COMORBILIDAD DE CHARLSON.}

\begin{tabular}{|c|c|}
\hline \multicolumn{2}{|c|}{ Por favor, marque la casilla asociada a cada patología, en caso que el paciente la padezca. } \\
\hline$\square$ IAM & 1 \\
\hline$\square$ Insuficiencia cardiaca & 1 \\
\hline$\square$ Enfermedad arterial periférica & 1 \\
\hline$\square$ Enfermedad cerebrovascular & 1 \\
\hline$\square$ Demencia & 1 \\
\hline$\square$ Enfermedad respiratoria crónica & 1 \\
\hline$\square$ Enfermedad del tejido conectivo & 1 \\
\hline$\square$ Úlcera gastroduodenal & 1 \\
\hline$\square$ Hepatopatía crónica leve & 1 \\
\hline$\square$ Diabetes sin lesión órganos diana & 1 \\
\hline$\square$ Hemiplejia & 2 \\
\hline$\square$ Insuficiencia renal crónica & 2 \\
\hline$\square$ Diabetes con lesión órganos diana & 2 \\
\hline$\square$ Tumor o neoplasia sólida sin metástasis & 2 \\
\hline$\square$ Leucemia & 2 \\
\hline$\square$ Linfoma & 2 \\
\hline$\square$ Hepatopatía crónica moderada/severa & 3 \\
\hline$\square$ Tumor o neoplasia sólida con metástasis & 6 \\
\hline$\square$ SIDA & 6 \\
\hline & \\
\hline
\end{tabular}


porcentaje importante de la muestra presentó otros síntomas urinarios del síndrome de $\mathrm{VH}$, siendo los más frecuentes la frecuencia miccional aumentada (mayor o igual a cada dos horas) y la incontinencia urinaria de urgencia (IUU), predominante en mujeres. Por otra parte los síntomas de dificultad miccional, con excepción del dolor, fueron más frecuentes en hombres que en mujeres.

En la Tabla $V$ se resumen los resultados del estudio de asociación de las diferentes variables personales y clínicas generales con la nocturia, de manera separada para ambos sexos. La mayor edad y la presencia de comorbilidades se asociaron con la nocturia tanto para hombres como para mujeres. En mujeres la menopausia está también asociada a la nocturia, mientras que estar sometida a un tratamiento hormonal sustitutivo o al tratamiento con estrógenos vaginales no se asocian significativamente a este síntoma.

Realizando un estudio de asociación similar para los síntomas urinarios (Tabla Vl) se encontró que en ambos sexos, los síntomas urinarios de urgencia, frecuencia e IUU fueron más frecuentes en pacientes con nocturia. En cuanto a los síntomas asociados a la dificultad miccional, sólo el tener que hacer fuerza para orinar se asoció con la nocturia en hombres. Por otro lado la variable dolor se asoció con la nocturia en mujeres.

En las Tablas VII y VIII se muestran modelos de regresión logística para la variable "nocturia", de manera separada para los subgrupos de mujeres y hombres respectivamente, que determinan la asociación independiente de las diferentes variables personales y clínicas no urinarias con la nocturia. En el subgrupo de mujeres, de los factores que habían mostrado asociación significativa en el estudio de asociación de variables aisladas, la menopausia no presentó asociación independiente con la nocturia. En el subgrupo de hombres tanto la edad como el índice de comorbilidad parecen influir de manera significativa en la predicción de la nocturia.

\section{DISCUSIÓN}

En el presente estudio, se describen las características del elevado porcentaje de pacientes con síntomas de $\mathrm{VH}$ que padecen el síntoma de nocturia $184,6 \%$ tenían dos o más micciones nocturnas; $81,4 \%$

TABLA III. DESCRIPCIÓN DE LA MUESTRA. VARIABLES DEMOGRÁFICAS Y CLÍNICAS.

\begin{tabular}{|c|c|c|c|c|c|}
\hline & & $\begin{array}{l}\text { Muestra total } \\
\qquad(\mathrm{N}=1.454)\end{array}$ & $\begin{array}{c}\text { Mujeres } \\
(\mathrm{N}=1.056)\end{array}$ & $\begin{array}{l}\text { Varones } \\
(\mathrm{N}=397)\end{array}$ & $\mathbf{p}$ \\
\hline Sexo & $\begin{array}{l}\text { Mujer } \\
\text { Hombre }\end{array}$ & $\begin{array}{l}72,6 \% \\
27,4 \%\end{array}$ & & & \\
\hline Edad media (D.T.) & & $60,1(12,3)$ & $58,5(12,3)$ & $64,3(11,4)$ & $<0,001$ \\
\hline Índice de Charlson & $\begin{array}{l}0 \\
1 \\
>=2\end{array}$ & $\begin{array}{l}46,9 \% \\
25,7 \% \\
27,4 \%\end{array}$ & $\begin{array}{l}53,7 \% \\
26,0 \% \\
20,3 \%\end{array}$ & $\begin{array}{l}28,9 \% \\
24,6 \% \\
46,5 \%\end{array}$ & $<0,001$ \\
\hline Partos vaginales & & & $85,2 \%$ & & \\
\hline Menopausia & & & $76,1 \%$ & & \\
\hline THS* & & & $8,9 \%$ & & \\
\hline Estrógenos vaginales & & & $11,7 \%$ & & \\
\hline
\end{tabular}

* tratamiento hormonal sustitutivo sistémico 
TABLA IV. DESCRIPCIÓN DE LA MUESTRA SÍNTOMAS URINARIOS.

\begin{tabular}{|l|c|c|c|c|}
\hline & $\begin{array}{c}\text { Muestra total } \\
(\mathbf{N}=1.454)\end{array}$ & $\begin{array}{c}\text { Mujeres } \\
(\mathbf{N}=1.056)\end{array}$ & $\begin{array}{c}\text { Varones } \\
(\mathbf{N}=398)\end{array}$ & \\
\hline Nocturia & & & & \\
O veces & $3,2 \%$ & $3,8 \%$ & $1,5 \%$ & $5,5 \%$ \\
Sólo 1 vez & $12,2 \%$ & $14,8 \%$ & 9,041 \\
\hline >= 2 veces & $84,6 \%$ & $81,4 \%$ & $93 \%$ & $<0,001$ \\
\hline Urgencia miccional & $78,5 \%$ & $79,5 \%$ & $75,6 \%$ & 0,105 \\
\hline Frecuencia & $70,1 \%$ & $69,1 \%$ & $72,6 \%$ & 0,219 \\
\hline Dificultad inicio & $26,7 \%$ & $20,4 \%$ & $43,5 \%$ & $<0,001$ \\
\hline Fuerza al orinar & $25,0 \%$ & $18,2 \%$ & $43,2 \%$ & $<0,001$ \\
Interrumpe chorro & $27,7 \%$ & $21,8 \%$ & $43,5 \%$ & $<0,001$ \\
\hline IU de urgencia & $71,7 \%$ & $75,0 \%$ & $62,8 \%$ & $<0,001$ \\
\hline IU de esfuerzo & $37,1 \%$ & $43,0 \%$ & $21,4 \%$ & $<0,001$ \\
\hline Dolor & $41,0 \%$ & $41,7 \%$ & $39,2 \%$ & 0,427 \\
\hline
\end{tabular}

* Significación estadística para las diferencias entre los grupos de hombres y mujeres.

TABLA V. ASOCIACIÓN DE LAS DIFERENTES VARIABLES PERSONALES Y CLÍNICAS CON LA NOCTURIA

\begin{tabular}{|c|c|c|c|c|c|c|c|}
\hline & \multicolumn{3}{|c|}{ Mujeres $(\mathrm{N}=1.056)$} & \multicolumn{3}{|c|}{ Varones $(\mathrm{N}=398)$} \\
\hline & & Sin nocturia & Con nocturia & $\mathbf{p}$ & Sin nocturia & Con nocturia & $\mathbf{p}$ \\
\hline Edad media (D.T.) & & $55(12,3)$ & $59,3(12,2)$ & $<0,001$ & $55,25(14)$ & $65(10,9)$ & $<0,001$ \\
\hline Índice de Charlson & 0 & $68,37 \%$ & $50,35 \%$ & & $60,71 \%$ & $26,49 \%$ & \\
\hline & 1 & $22,45 \%$ & $26,86 \%$ & $<0,001$ & $38,89 \%$ & $24,59 \%$ & $<0,001$ \\
\hline & $>=2$ & $9,18 \%$ & $22,79 \%$ & & $14,29 \%$ & $48,92 \%$ & \\
\hline Partos vaginales & & $81,12 \%$ & $86,16 \%$ & 0,092 & & & \\
\hline Menopausia & & $68,37 \%$ & $77,86 \%$ & 0,007 & & & \\
\hline Con THS* & & $10,20 \%$ & $8,60 \%$ & 0,568 & & & \\
\hline Estrógenos vaginales & & $7,65 \%$ & $12,67 \%$ & 0,065 & & & \\
\hline
\end{tabular}

* tratamiento hormonal sustitutivo sistémico 
de las mujeres y $93 \%$ de los varones). La edad y la comorbilidad medida por el Índice de Charlson se asocian de manera independiente con la presencia del síntoma nocturia tanto en varones como en mujeres.

El estudio muestra cómo en pacientes previamente diagnosticados de $\mathrm{VH}$, la prevalencia de la nocturia se sitúa en el $84,6 \%$ considerando 2 o más micciones nocturnas, y en el $96,8 \%$ aplicando la definición de la ICS. Estos porcentajes son claramente superiores a los mostrados en un estudio basado en una encuesta telefónica en 5 países (18), que mostró una prevalencia de la nocturia en sujetos con $\mathrm{VH}$ del $75 \%$ utilizando la definición de la ICS (sólo un $40 \%$ aplicando el criterio más estricto). Estas diferencias pueden deberse, además de las diferencias inherentes a las diferentes poblaciones de estudio, a que en el presente estudio se basa en pacientes clínicamente diagnosticados de $\mathrm{VH}$ mientras que el reportado se basa en la respuesta telefónica afirmativa de la población general a preguntas sobre urgencia e IUU. La elevada prevalencia de la nocturia en pacientes con $\mathrm{VH}$ pone de manifiesto la importancia de la identificación de la nocturia en estos pacientes para su

TABLA VI. ASOCIACIÓN DE SÍNTOMAS URINARIOS CON EL SÍNTOMA NOCTURIA, SEPARADO POR SEXO.

\begin{tabular}{|c|c|c|c|c|c|c|c|c|c|}
\hline & & \multicolumn{4}{|c|}{ Mujeres } & \multicolumn{4}{|c|}{ Varones } \\
\hline Variable & & $\begin{array}{c}\text { Sin } \\
\text { Nocturia* }\end{array}$ & $\begin{array}{c}\text { Con } \\
\text { Nocturia* }\end{array}$ & $\begin{array}{c}\text { Chi } \\
\text { cuadrado }\end{array}$ & $p$ & $\begin{array}{c}\text { Sin } \\
\text { Nocturia* }\end{array}$ & $\begin{array}{c}\text { Con } \\
\text { Nocturia* }\end{array}$ & $\begin{array}{c}\text { Chi } \\
\text { cuadrado }\end{array}$ & $\mathbf{p}$ \\
\hline Urgencia & $\begin{array}{l}\text { No } \\
\text { Sí }\end{array}$ & $\begin{array}{l}39,35 \\
13,21\end{array}$ & $\begin{array}{l}60,65 \\
86,79\end{array}$ & 75,93 & $<0,001$ & $\begin{array}{l}17,53 \\
3,65\end{array}$ & $\begin{array}{l}82,47 \\
96,35\end{array}$ & 19,51 & $<0,001$ \\
\hline Frecuencia & $\begin{array}{l}\text { No } \\
\text { Sí }\end{array}$ & $\begin{array}{l}32,82 \\
12,19\end{array}$ & $\begin{array}{l}67,18 \\
87,81\end{array}$ & 62,1 & $<0,001$ & $\begin{array}{c}17,43 \\
3,11\end{array}$ & $\begin{array}{l}82,57 \\
96,89\end{array}$ & 22,67 & $<0,001$ \\
\hline Dificultad & $\begin{array}{l}\text { No } \\
\text { Sí }\end{array}$ & $\begin{array}{l}18,79 \\
17,67\end{array}$ & $\begin{array}{l}81,21 \\
82,33\end{array}$ & 0,076 & 0,782 & $\begin{array}{l}8,89 \\
4,62\end{array}$ & $\begin{array}{l}91,11 \\
95,38\end{array}$ & 2,107 & 0,147 \\
\hline Fuerza & $\begin{array}{l}\text { No } \\
\text { Sí }\end{array}$ & $\begin{array}{l}18,63 \\
18,23\end{array}$ & $\begin{array}{l}81,37 \\
81,77\end{array}$ & 0,001 & 0,978 & $\begin{array}{l}10,18 \\
2,91\end{array}$ & $\begin{array}{l}89,82 \\
97,09\end{array}$ & 6,82 & 0,009 \\
\hline Interrumpe & $\begin{array}{l}\text { No } \\
\text { Sí }\end{array}$ & $\begin{array}{l}18,77 \\
17,83\end{array}$ & $\begin{array}{l}81,23 \\
82,17\end{array}$ & 0,052 & 0,82 & $\begin{array}{l}8,00 \\
5,78\end{array}$ & $\begin{array}{l}92,00 \\
94,22\end{array}$ & 0,436 & 0,509 \\
\hline IUU & $\begin{array}{l}\text { No } \\
\text { Sí }\end{array}$ & $\begin{array}{l}32,95 \\
13,76\end{array}$ & $\begin{array}{l}67,05 \\
86,24\end{array}$ & 46,99 & $<0,001$ & $\begin{array}{l}12,16 \\
4,00\end{array}$ & $\begin{array}{l}87,84 \\
96,00\end{array}$ & 8,26 & 0,004 \\
\hline IUE & $\begin{array}{l}\text { No } \\
\text { Sí }\end{array}$ & $\begin{array}{l}19,93 \\
16,74\end{array}$ & $\begin{array}{l}80,07 \\
83,26\end{array}$ & 1,54 & 0,214 & $\begin{array}{l}7,67 \\
4,71\end{array}$ & $\begin{array}{l}92,33 \\
95,29\end{array}$ & 0,5 & 0,479 \\
\hline Dolor & $\begin{array}{l}\text { No } \\
\text { Sí }\end{array}$ & $\begin{array}{l}22,73 \\
12,73\end{array}$ & $\begin{array}{l}77,27 \\
87,27\end{array}$ & 16,98 & $<0,001$ & $\begin{array}{l}7,85 \\
5,77\end{array}$ & $\begin{array}{l}92,15 \\
94,23\end{array}$ & 0,351 & 0,554 \\
\hline
\end{tabular}

* \% observados para cada uno de los casos. 


\section{TABLA VII. MODELO DE REGRESIÓN LOGISTICA PARA DETERMINAR LAS VARIABLES ASOCIADAS INDEPENDIENTEMENTE CON LA NOCTURIA EN MUJERES.}

\begin{tabular}{|l|c|c|c|c|c|}
\hline Variable & B & Exp(B) & IC(95\%) & Wald & p \\
\hline Constante & 0,041 & 1,042 & & 0,009 & 0,926 \\
\hline Edad & 0,024 & 1,024 & $1,004-1,045$ & 5,525 & 0,019 \\
\hline Charlson (0, 1, >=2) & & & & 13,305 & 0,001 \\
\hline 1 & 0,367 & 1,443 & $0,976-2,133$ & 3,377 & 0,066 \\
\hline$>=2$ & 0,990 & 2,693 & $1,551-4,671$ & 12,397 & $<0,001$ \\
\hline
\end{tabular}

Modelo ajustado para las variables personales y clínicas no urinarias previamente asociadas a la nocturia en mujeres (Edad, Menopausia y Comorbilidades) $R^{2}=0,053$.

tratamiento específico, y más aún si se tiene en cuenta que la combinación de ambas sintomatologías impacta de manera importante en la calidad de vida del paciente.

Los síntomas urinarios más frecuentes en la muestra además de la urgencia, inherente a la $\mathrm{VH}$, fueron la frecuencia $(70,1 \%)$ y la IUU $(71,7 \%)$, que se presentó con mayor frecuencia en mujeres. Los síntomas asociados a la dificultad miccional así como la IUE también presentaron una distribución significativamente diferente en varones y mujeres. Por otra parte, los síntomas urinarios de frecuencia, urgencia e IUU se asociaron con la nocturia en ambos sexos, mientras que la IUE no mostró asociación, la variable "dolor" se asoció solo en mujeres y la variable de dificultad miccional "fuerza" sólo en varones. Los síntomas urinarios no fueron incluidos en el análisis de regresión logística posterior ya que se considera que estos y la nocturia podrían depender de una causa común, lo que haría difícil la interpretación de su asociación independiente.

En nuestro estudio se muestra además que parámetros personales y clínicos generales como una mayor edad y el índice de comorbilidad se asocian tanto de forma bivariada como independientemente en modelos de regresión logística a la presencia de nocturia en pacientes con $\mathrm{VH}$. El aumento de la prevalencia de la nocturia con la edad había sido previamente descrito en estudios anteriores (8-10). En un estudio poblacional en Dinamarca se observó

\section{TABLA VIII. MODELO DE REGRESIÓN LOGÍSTICA PARA DETERMINAR LAS VARIABLES ASOCIADAS INDEPENDIENTEMENTE CON LA NOCTURIA EN VARONES.}

\begin{tabular}{|l|c|c|c|c|c|}
\hline Variable & B & $\operatorname{Exp(B)}$ & $\mathbf{I C ( 9 5 \% )}$ & Wald & $\mathbf{p}$ \\
\hline Constante & $-0,439$ & 0,645 & & 0,241 & 0,645 \\
\hline Edad & 0,040 & 1,041 & $1,008-1,074$ & 6,051 & 0,014 \\
\hline Charlson (0,1,>=2) & & & & 6,272 & 0,043 \\
\hline 1 & 0,564 & 1,758 & $0,673-4,593$ & 1,325 & 0,250 \\
\hline$>=2$ & 1,530 & 4,616 & $1,376-15,490$ & 6,133 & 0,013 \\
\hline
\end{tabular}

Modelo ajustado para las variables personales y clínicas no urinarias previamente asociadas a la nocturia en hombres (Edad y Comorbilidades) $R^{2}=0,142$. 
que la edad se asociaba de forma significativa a la nocturia, independientemente de la gravedad de la misma (19).

En la submuestra de mujeres la menopausia no apareció en el modelo regresión como parámetro independiente significativamente predictivo de nocturia, mientras que si se asoció a la misma en el análisis bivariado. Este resultado contrasta con los mostrados en un estudio anterior que indicaban una asociación independiente de la edad y la menopausia en la predicción de la nocturia en pacientes con IU (20). Esto podría deberse a la mayor edad media y mayor frecuencia de menopausia del presente estudio, lo cual haría perder peso al factor menopausia como factor independiente, siendo en realidad el efecto de la edad lo que actuaría como factor de riesgo.

Una posible limitación de este estudio radica en que la muestra objeto de estudio presenta el sesgo de que corresponde a pacientes que acudieron a una consulta especializada de urología o ginecología, y no sería en principio extrapolable al conjunto de la población que sufre $\mathrm{VH}$, sintomatología claramente infra-diagnosticada.

La nocturia es un síntoma urinario común y con un importante impacto en pacientes con $\mathrm{VH}$, que a menudo no se identifica como un problema en sí mismo. La presencia de nocturia en pacientes con $\mathrm{VH}$ se asocia a niveles elevados de molestia y una menor calidad de vida (14,21-22). Resulta lógico que la acumulación de sintomatologías urinarias haga que los pacientes tengan una menor tolerancia hacia los trastornos provocados por la nocturia. La correcta detección del trastorno en sí mismo, así como la identificación de sus causas específicas y la corrección de éstas en la medida de lo posible es de gran importancia para mejorar la calidad de vida de los pacientes, afectada sobre todo por la disminución de la calidad del sueño que deriva en un amplio espectro de morbilidades y trastornos asociados.

\section{CONCLUSIONES}

En este estudio se ha determinado que en pacientes previamente diagnosticados de $\mathrm{VH}$ el perfil de los sujetos que presentan nocturia responde a una mayor edad y a la presencia de comorbilidades de cierta importancia (charlson $>=2$ ) tanto para varones como para mujeres. Esta descripción es novedosa y pretende contribuir a la adecuada identificación y manejo de pacientes con nocturia. Por otra parte, el perfil de nocturia en pacientes con $\mathrm{VH}$ presentado difiere de los reportados para pacientes con otras patologías urinarias como se ha resaltado anteriormente.

\section{BIBLIOGRAFÍA y LECTURAS RECOMENDADAS ( ${ }^{*}$ lectura de interés $y^{* *}$ lectura fundamental)}

*1. Abrams P, Cardozo L, Fall M, Griffiths D, Rosier P, Ulmsten U et al.; Standardisation Sub-Committee of the International Continence Society. The standardisation of terminology in lower urinary tract function: report from the standardisation sub-committee of the International Continence Society. Urol, 2003; 61: 37-49.

*2. Castro D, Espuña M, Prieto M, Badia X. Prevalence of overactive bladder in Spain: a population-based study. Arch Esp Urol, 2005; 58:131-8.

3. Van Kerrebroeck P. Standardization of terminology in nocturia: commentary on the ICS report. BJU Int 2002; 90:16-17.

4. Grupo Español de Urodinámica y de la SINUG. Consensus over terminology and concepts for lower urinary tract function. Actas Urol Esp 2005; 29:16-30.

5. Swithinbank LV, Donovan J, James MC, Yang Q, Abrams P. Female urinary symptoms: age prevalence in a community dwelling population using a validated questionnaire. Neurol Urodyn 1998; 16:432-4.

6. Mattsson S. Urinary incontinence and nocturia in healthy schoolchildren. Acta Paediatr 1994; 83:950-4.

*7. Middelkoop HA, Smilde-van den Doel DA, Neven AK, Kamphuisen HA, Springer CP. Subjective sleep characteristics of 1,485 males and females aged 50-93: effects of sex and age, and factors related to self-evaluated quality of sleep. J Gerontol A Biol Sci Med Sci 1996; 51:108-15.

8. Schatzl G, Temml C, Schmidbauer J, Dolezal B, Haidinger G, Madersbacher S. Cross-sectional study of nocturia in both sexes: analysis of a voluntary health screening project. Urology 2000; 56:71-5.

9. Coyne KS, Zhou Z, Bhattacharyya SK, Thompson CL, Dhawan R, Versi E. The prevalence of nocturia and its effect on health-related quality of life and sleep in a community sample in the USA. BJU Int 2003; 92: 948-54.

10. Yoshimura K, Terada N, Matsui Y, Terai A, Kinukawa N, Arai Y. Prevalence of and risk factors for nocturia: Analysis of a health screening program. Int J Urol 2004; 11:282-7.

11. Wennberg AL, Molander U, Fall M, Edlund C, Peeker R, Milsom I. A Longitudinal Populationbased Survey of Urinary Incontinence, Overactive Bladder, and Other Lower Urinary Tract Symptoms in Women. Eur Urol 2009; 55: 783-91.

*12. Irwin DE, Milsom I, Hunskaar S, Reilly K, Koop $\mathrm{Z}$, Herschorn S, et al. Population-based survey of urinary incontinence, overactive bladder, and 
other lower urinary tract symptoms in five countries: results of the EPIC study. Eur Urol 2006; 50: 1306-15.

*13. Desgrandchamps F, Cortesse A, Rousseau T, Teillac P, Duc AL. Normal voiding behaviour in women. Study of the I-PSS in an unselected population of women in general practice. Eur Urol 1996; 30: 18-23.

**14. Coyne KS, Zhou Z, Bhattacharyya SK, Thompson CL, Dhawan R, Versi E. The prevalence of nocturia and its effect on health-related quality of life and sleep in a community sample in the USA. BJU Int 2003; 92: 948-54.

15. Jackson S, Donovan J, Brookes S, Eckford S, Swithinbank L, Abrams P. The Bristol female lower urinary tract symptoms questionnaire: Development and psychometric testing. Br J Urol 1996; 77: 805-12.

16. Donovan JL, Abrams P, Peters TJ, Kay HE, Reynard J, Chapple C, et al. The ICS-'BPH' Study: the psychometric validity and reliability of the ICSmale questionnaire. Br J Urol 1996; 77: 554-62.

17. Charlson ME, Pompei P, Ales KL, MacKenzie CR. A new method of classifying prognostic co- morbidity in longitudinal studies: development and validation. J Chronic Dis 1987; 40: 373-83.

**18. Irwin DE, Abrams P, Milsom I, Kopp Z, Reilly K; EPIC Study Group. Understanding the elements of overactive bladder: questions raised by the EPIC study. BJU Int 2008; 101:1381-7.

**19. Bing MH, Moller LA, Jennum P, Mortensen S, Lose G. Nocturia and associated morbidity in a Danish population of men and women aged 60-80 years. BJU Int 2008; 102:808-14.

**20. Espuña Pons M, Puig Clota M, Pérez González A, Rebollo Álvarez A. Nicturia en mujeres con síntomas de incontinencia urinaria: análisis de las variables clínicas y urodinámicas asociadas. Actas Urol Esp 2005; 29: 378-86.

**21. Coyne KS, Payne C, Bhattacharyya SK, Revicki DA, Thompson C, Corey R, et al. The impact of urinary urgency and frequency on health-related quality of life in overactive bladder: results from a national community survey. Value Health 2004; 7: 455-63.

**22. Abrams P. Nocturia: The effects on sleep and related health consequences. Eur Urol Suppl 2005; 3: 8-16. 